\title{
CÁC NHÂN TỐ ẢNH HƯởNG ĐẾN TỔ CHỨC Bộ PHẬN KIỂM TOÁN NỘI BỘ TẠI CÁC TRƯờnG ĐẠI HỌC CÔNG LẬP THEO CƠ CHẾ TƯं CHỦ VIÊTT NAM - NGHIÊN CÚU TİNH HUỐNG TẠI TRƯỜnG ĐẠI HỌC CÔNG NGHIỆP TP.HCM
}

\author{
TĂNG THI THANH THỦY - CAO THI CÂMM VÂN \\ Truờng Đại học Công nghiệp Thành phố Hồ Chí Minh \\ tangthithanhthuy@iuh.edu.com
}

\begin{abstract}
Tóm tắt: Nghiên cứu này được thực hiện với mục tiêu khám phá các nhân tố ảnh hưởng đến tổ chức bộ phận kiểm toán nội bộ (KTNB) tại các trường Đại học công lập theo cơ chế tự chủ Việt Nam nói chung và trường Đại học Công nghiệp TP.HCM nói riêng. Phương pháp phân tích Crombach 's Alpha, phân tích EFA cùng với phân tích hồi quy đa biến được chúng tôi sử dụng với phương tiện SPSS. Kết quả nghiên cứu cho thấy có 4 nhân tố ảnh hưởng cùng chiều đến tổ chức Bộ phận KTNB tại các trường Đại học công lập theo cơ chế tự chủ Việt Nam gồm có: mô hình tổ chức bộ phận KTNB, năng lực chuyên môn, sự hỗ trợ của nhà quản lý trong tổ chức Bộ phận KTNB và phạm vi công việc của KTNB.
\end{abstract}

Từ khóa: Bộ phận kiểm toán nội bộ, kiểm toán nội bộ, Đại học công lập theo cơ chế tự chủ.

\section{FACTORS AFFECTS ESTABLISHMENT OF INTERNAL AUDITING DEPARTMENT IN THE VIETNAMESE PUBLIC UNIVERSITIES}

\begin{abstract}
This study aims to explore factors affecting the organization of the Internal Audit Department at the Vietnamese public universities under autonomy system and Industrial University of Ho Chi Minh City in particular. The method of Crombach 's Alpha analysis, EFA analysis and multiple regression analysis were used with the SPSS program. The result shows that four factors affect such as organizational structures, competence of Internal Audit Department, management support to Internal Audit Department, and scope of Internal Audit's work
\end{abstract}

Key words: Internal audit department, Internal audit function, public university

\section{GIỚI THIỆ}

Kiểm toán nội bộ (KTNB) là một chức năng độc lập được thiết lập nhằm kiểm tra, đánh giá hoặc tư vấn để đưa ra các đảm bảo và kiến nghị về hoạt động tổ chức, hỗ trợ gia tăng giá trị cho tổ chức (IIA, 2017). Sự hiện diện của Bộ phận KTNB trong các tổ chức được xem như là "bức tường lửa" bảo vệ duy trì hệ thống quản trị tổ chức tốt, trợ giúp Ban quản lý đánh giá và thiết lập được các quy trình kiểm soát rủi ro.

Tại Việt Nam, kể từ sau khi Bộ Tài Chính ban hành quy chế kiểm toán nội bộ theo quyết định 832/TC/QĐ/CĐKT, hoạt động KTNB đã có sự phát triển nhất định trong các tổ chức kinh tế. Tuy nhiên, hoạt động này gần như chưa tồn tại trong các trường Đại học công lập Việt Nam. Trong bối cảnh được trao quyền tự chủ theo Nghị quyết 77/NQ-CP, các trường Đại học này phải đối mặt với những áp lực cao hơn trước khi thực hiện các quy định của nhà nước. Vì vậy, sự hiện diện của một cơ chế giám sát hiệu quả là cần thiết cho các các trường Đại học hiện nay. Theo xu thế phát triển của quản trị hiện đại và kinh nghiệm của các trường Đại học danh tiếng trên thế giới, Kiểm toán nội bộ được xem là lựa chọn phù hợp nhất cho cơ chế giám sát này.

Trường Đại học Công nghiệp Thành phố Hồ Chí Minh (TP.HCM) là Trường Đại học công lập được nâng cấp từ Trường Cao đẳng Công nghiệp IV theo quyết định số 214/2004/QĐ - TTg của Thủ tướng Chính phủ. Từ năm học 2015 - 2016, trường là một trong những cơ sở giáo dục đại học lớn tại Việt Nam được Thủ tưởng Chính phủ phê duyệt thí điểm đổi mới cơ chế hoạt động tự chủ theo quyết định số 902/QĐ-TTg ngày 23 tháng 6 năm 2015. Trường có đội ngũ cán bộ - giảng viên là 1.439 người, trong đó có 1.111 giảng viên cơ hữu. Về cơ cấu tổ chức nhà trường được hình thành khá đa dạng gồm có: Ban 
giám hiệu, các Hội đồng tư vấn và đoàn thể, 16 phòng ban chức năng và 20 khoa giảng dạy chuyên ngành. Bên cạnh cung cấp dịch vụ đào tạo, nhà trường còn kinh doanh hoạt động xuất bản và sản xuất nước uống tinh khiết đóng chai. Hiện tại, trường có 01 cơ sở chính tại TP.HCM, 01 phân hiệu Quảng Ngãi và 01 cơ sở Thanh Hóa. Trường luôn chú trọng nâng cao chất lượng chuyên môn và cung cấp các dịch vụ đào tạo có chất lượng cao cho hàng chục ngàn sinh viên từ khắp mọi miền trong cả nước. Với quy mô lớn và hoạt động kinh doanh đa dạng cùng với định hướng của nhà trường là tiếp cận theo chuẩn quốc tế, nhu cầu xây dựng một cơ chế giám sát để nâng cao chất lượng và hỗ trợ Ban quản lý nhà trường trong việc ngăn ngừa các rủi ro, tăng giá trị cho nhà trường như Bộ phận KTNB là cần thiết.

Do đó, trong bài báo này, chúng tôi tập trung nghiên cứu các nhân tố ảnh hưởng đến tổ chức Bộ phận KTNB nhằm có định hướng xây dựng hoạt động kiểm toán nội bộ hiệu quả cho các trường Đại học công lập theo cơ chế tự chủ nói chung và trường Đại học Công nghiệp TP.HCM nói riêng.

\section{TỔNG QUAN CƠ SỞ LÝ THUYẾT VÀ CÁC NGHIÊN CÚU TRƯớC}

Để giải thích cho sự ra đời và phát triển KTNB, Elliott (1992) dựa vào lý thuyết cung và cầu được đề xuất bởi Watts et al (1983) đã chứng minh rằng trong điều kiện của cuộc "cách mạng thông tin" vào cuối thế kỷ 20 , người sử dụng cần được cung cấp thông tin về hoạt động của đơn vị một cách liên tục. Chính vì vậy, cần có sự phát triển của nghề nghiệp kiểm toán mới như kiểm toán nội bộ để đáp ứng nhu cầu này.

Theo lý thuyết đại diện, trong một tổ chức luôn tồn tại mâu thuẫn lợi ích cá nhân giữa nhà quản lý và chủ sở hữu (Jensen và Meckling, Adam, 1994). Lý thuyết bất đối xứng thông tin (Akerlof, 1970) cho rằng nhà quản lý thường có nhiều thông tin hữu ích hơn các chủ sở hữu. Tuy nhiên, theo lý thuyết các bên liên quan được giới thiệu lần đầu bởi Freeman (1984), các nhà quản lý tổ chức phải tìm cách để hòa hợp lợi ích của các bên liên quan. Lý thuyết tín hiệu lần đầu tiên được công bố bởi Spence (1973) cũng đề xướng giải pháp nhằm giảm hậu quả của tình trạng thông tin bất cân xứng bằng cách bên có nhiều thông tin có thể chủ động thực hiện "phát tín hiệu" nhằm thuyết phục các bên (thiếu thông tin). Bên cạnh đó, lý thuyết hợp pháp hóa cũng yêu cầu các đơn vị phải luôn đảm bảo hoạt động hợp pháp và nằm trong phạm vi, quy chuẩn thay đổi không ngừng theo kỳ vọng của xã hội. Nhìn chung, xét trên phương diện quản trị tổ chức, các lý thuyết trên đều nhấn mạnh đến nhu cầu thông tin minh bạch và giải thích lý do các đơn vị cần xây dựng cơ chế giám sát hiệu quả như Bộ phận KTNB để cung ứng được thông tin có độ tin cậy cao cho các bên liên quan đưa ra quyết định phù hợp.

Trong vài thập kỷ gần đây khá nhiều nghiên cứu về hoạt động KTNB đều thừa nhận Bộ phận KTNB là một phần quan trọng không thể thiếu trong cấu trúc hoạt động của một tổ chức (Donna Jane Reed, 1999; Al-Twaijry et al, 2003; Goodwin, 2004; Coram et al, 2008; Arena et al, 2009). Đặc biệt, sau khi Đạo luật $\mathrm{SOX}$ được ban hành năm 2002, vai trò của KTNB ngày càng nâng cao nhằm gia tăng giá trị và tăng cường khả năng cạnh tranh cho tổ chức (Hass et al, 2006; Allegrini et al, 2006; Coopers et al, 2006). Thực hiện nghiên cứu tương tự, Wallace et al (1991) còn nhấn mạnh tổ chức Bộ phận KTNB phụ thuộc nhiều vào các quy định pháp lý, quy mô của tổ chức, mối quan hệ với kiểm toán độc lập, sự hiện diện của Ban kiểm soát và áp lực phải đạt được mục tiêu kinh doanh. Mặt khác, nghiên cứu của Carey et al., (2000); Goodwin (2004) cũng chứng minh rằng gần đây các đơn vị thường có khuynh hướng sử dụng KTNB thuê ngoài. Tuy nhiên, nghiên cứu của Kalbers et al (1993), Quales (1994), Fraser et al (2007), Barac et al (2009) khẳng định các đơn vị sử dụng KTNB thuê ngoài sẽ không đạt được hiệu quả kiểm soát cao, đe dọa tính độc lập cũng như khả năng bảo mật thông tin so với KTNB bên trong.

Tổ chức Bộ phận KTNB là cần thiết tuy nhiên để xác định được mô hình và quy mô Bộ phận KTNB phù hợp, các trường Đại học cần dựa vào đặc điểm cơ chế quản lý và hoạt động của đơn vị. Liu et al (1997), Arena et al (2009) khi nghiên cứu về mô hình tổ chức Bộ phận KTNB khẳng định rằng có bốn mô hình tổ chức Bộ phận KTNB thưởng được áp dụng gồm có mô hình tập trung, mô hình phân tán, mô hình ủy quyền và mô hình kết hợp.

Nghiên cứu của Anderson et al (2010) tập trung vào quy mô Bộ phận KTNB cũng đã chứng minh rằng có 7 nhóm nhân tố ảnh hưởng đến việc xác định quy mô phù hợp cho Bộ phận KTNB bao gồm: 1) đặc điểm của tổ chức, 2) đặc điểm cơ cấu quản trị tổ chức, 3) chức năng của bộ phận KTNB, 4) mức độ thực thi các đề xuất của $K T N B, 5)$ mối quan hệ giữa Bộ phận KTNB với Ban quản lý và Ủy ban kiểm toán, 6) cơ chế tuyển dụng cho Bộ phận KTNB và 7) chất lượng dịch vụ KTNB cung cấp. 
Nghiên cứu của Powell (1993); Dirsmith et al., (2000) thì nhấn mạnh đến hình thức tổ chức Bộ phận KTNB theo quy mô nhóm và cho rằng hình thức tổ chức này sẽ cung cấp nhiều thông tin đa dạng. Kết quả nghiên cứu của chúng tôi cũng cho thấy quy mô nhóm KTNB sẽ phụ thuộc vào loại hình kinh doanh, mức độ phức tạp của hoạt động kinh doanh, sự thay đổi và những khó khăn khi thực hiện các nhiệm vụ trong tổ chức. Ngược lại với quan điểm của Dirsmith et al., (2000), nghiên cứu của Firth-Cozens (1992) dựa trên phương pháp điều tra khảo sát lại cho rằng nhóm kiểm toán có nhiều nhân viên sẽ đe dọa đến tính hiệu quả công việc và sự bảo mật thông tin. Cho nên tác giả đề nghị nhóm kiểm toán tối đa chỉ gồm ba nhân viên thay vì bảy nhân viên như đề nghị trong các nghiên cứu trước. Thực hiện nghiên cứu tương tự tại Malaysia, Fadzil et al. (2005) cũng cung cấp bằng chứng đa số Bộ phận KTNB Malaysia $(57,6 \%)$ chỉ có 5 nhân viên và thường được tổ chức theo nhóm.

Nghiên cứu của Mihret (2009), Albercht et al (1988), Al-Twaijry et al (2003), Sarens et al (2006) thì tập trung vào sự hỗ trợ của nhà quản lý trong tổ chức Bộ phận KTNB. Kết quả nghiên cứu cho thấy sự hỗ trợ này thường được thực hiện thông qua các chương trình đào tạo để tăng cường kỹ năng kiểm toán (James và Mula, 2010; Engle, 1999) và nguồn ngân sách hoạt động dành cho KTNB để có thể tuyển dụng, duy trì được nguồn nhân sự KTNB chất lượng cao (Muller-Peters et al, 2001), đồng thời hỗ trợ kinh phí hoạt động cho Bộ phận này hoàn thành tốt chức năng được giao.

Mặc dù nhu cầu và cách thức tổ chức Bộ phận KTNB tại các quốc gia có điểm khác biệt nhưng các nhà nghiên cứu đều nhấn mạnh rằng cần xây dựng và phát triển nguồn nhân sự KTNB phù hợp, mang tính ổn định về mặt dài hạn bằng cách tập trung vào năng lực chuyên môn, sự độc lập khách quan và phạm vi hoạt động của KTNB (Messier and Scheiner, 1988; Al-Twaijry et al, 2003; Fadzil et al., 2005; Hajiha Zohreh và Rafiee Azadeh, 2011)

Nghiên cứu của Messier and Scheiner (1988) và Ashton (1991) về đặc điểm nguồn nhân sự trong tổ chức Bộ phận KTNB cho thấy năng lực chuyên môn của KTNB được đo lường bằng kinh nghiệm, chương trình đào tạo và chế độ giám sát hoạt động KTNB. Trong khi đó, nghiên cứu của Gramling and Myers (1997, 2006), Van Staden et al, (2009) thông qua khảo sát đã chứng minh được bằng cấp chuyên môn của KTV nội bộ, cụ thể là chứng chỉ nghề nghiệp được xem là một yếu tố để đo lường năng lực chuyên môn của KTNB khi tổ chức Bộ phận này. Nghiên cứu của Messier and Scheiner (1988) còn thừa nhận rằng phạm vi công việc được đo lường bằng phạm vi kiểm toán, kỹ năng kiểm toán, số lượng và chất lượng hệ thống giấy tờ làm việc cũng ảnh hưởng đến việc tổ chức Bộ phận KTNB.

Bên cạnh năng lực chuyên môn, nguồn nhân sự Bộ phận KTNB còn phải đảm bảo sự khách quan khi thực hiện công việc (IIA, 2017). Khá nhiều nghiên cứu cho rằng Bộ phận KTNB sẽ khách quan nếu được tự do phát biểu ý kiến mà không gặp phải sự can thiệp nào (Clark et al.,1980; Sawyer, 1988; Chapman, 2001; Yee et al., 2008, IIA, 2017). Do vậy, trưởng Bộ phận KTNB cần được bổ nhiệm bởi Hội đồng quản lý cấp cao của đơn vị để không gặp bất kỳ "sự lo ngại” nào khi thực hiện công việc kiểm toán (Chapman, 2001; Read and Rama, 2003). Trưởng Bộ phận KTNB cũng được yêu cầu phải báo cáo với nhà quản lý điều hành và Ban kiểm soát. Cơ chế báo cáo này sẽ tạo ra một chức năng kiểm toán khách quan để tăng niềm tin cho những người sử dụng kết quả công việc kiểm toán, ngăn chặn sự xung đột về lợi ích và sự thông đồng trong tổ chức (Cohen et al., 2004; DeZoort et al., 2002; Haron et al., 2005; Mat Zain \& Subramaniam, 2007; Vanasco, 1994, IIA, 2017).

Nghiên cứu của Arena et al (2009) điều tra việc chấp nhận và đặc điểm nguồn nhân sự Bộ phận KTNB tại Ý. Căn cứ vào dữ liệu khảo sát thu thập, chúng tôi cũng cho thấy nguồn nhân sự KTNB được đo lường bởi số lượng kiểm toán viên nội bộ, chương trình đào tạo hàng năm, kinh nghiệm làm việc, khuynh hướng nghề nghiệp, hồ sơ năng lực của Bộ phận KTNB. Bên cạnh đó, theo chúng tôi việc chấp nhận thành lập Bộ phận KTNB phụ thuộc vào đặc điểm đơn vị, mô hình tổ chức Bộ phận KTNB và các nhân tố bên ngoài tổ chức như quy định của nhà nước, các tổ chức nghề nghiệp đều ảnh hưởng đến việc tổ chức Bộ phận này. 

TẠI TRUUỜNG ĐẠI HỌC CỔNG NGHIỆP TP.HCM

\section{MÔ HİNH NGHIÊN CỨU VÀ PHƯƠNG PHÁP NGHIÊN CỨU}

\subsection{Mô hình nghiên cứu}

Kế thừa từ tổng quan các nghiên cứu trước và kết quả nghiên cứu định tính, chúng tôi xác định được 01 biến phụ thuộc là Tổ chức Bộ phận KTNB tại các trường Đại học công lập theo cơ chế tự chủ Việt Nam (IAR) và 6 biến độc lập gồm có:

- IM: là biến đại diện cho mô hình tổ chức Bộ phận KTNB. Biến IM thể hiện cách thức tổ chức và quy mô hoạt động của Bộ phận KTNB. Bộ phận này có thể trực thuộc sự quản lý của Ban giám hiệu hoặc Ban kiểm soát nhà trường. Quy mô Bộ phận KTNB cũng phụ thuộc vào tỷ lệ giữa số lượng KTV nội bộ trên số lượng nhân viên đơn vị. Biến IM dựa trên nghiên cứu của Allegrini et al., (2006); Arena et al., (2009).

- IS: là biến đại diện cho quy mô Bộ phận KTNB. Biến này thể hiện việc xác định quy mô của Bộ phận KTNB phụ thuộc vào đặc điểm của tổ chức cũng như nhiệm vụ, chức năng của Bộ phận này. Để đạt được sự hiệu quả trong thực hiện kiểm toán, Bộ phận KTNB thường được tổ chức theo quy mô nhóm. Biến IS dựa trên nghiên cứu của Anderson et al., (2010); Dirsmith et al., (2000) và Powell (1993).

- SM: là biến đại diện cho sự hỗ trợ của nhà quản lý trong tổ chức Bộ phận KTNB. Biến này thể hiện nguồn ngân sách mà nhà quản lý đầu tư cho việc tuyển dụng và hoạt động của Bộ phận này. Sự hỗ trợ của nhà quản lý còn thể hiện thông qua việc thực hiện các chương trình đào tạo và chế độ đãi ngộ dành cho nhân viên KTNB để nâng cao năng lực chuyên môn và trách nhiệm của họ. Biến SM dựa trên nghiên cứu của Mihret (2009); Albercht et al., (1988); Al-Twaijry et al., (2003); Sarens et al., (2006).

- IA: là biến đại diện năng lực chuyên môn của Bộ phận KTNB. Biến này thể hiện qua việc cần phải tổ chức Bộ phận KTNB gồm có những nhân viên có kinh nghiệm, bằng cấp nghề nghiệp và được tham gia chương trình cập nhật kiến thức hàng năm. Năng lực chuyên môn tốt sẽ nâng cao tính hiệu quả của Bộ phận KTNB trong việc hoàn thành nhiệm vụ được giao. Biến IA dựa trên nghiên cứu của Messier and Scheiner (1988); Gramling và Myers (1997, 2006); Van Staden et al., (2009).

- $\quad \mathrm{OB}$ : là biến đại diện tính khách quan của Bộ phận KTNB. Biến OB thể hiện Bộ phận KTNB cần được tổ chức độc lập, không có quan hệ với các phòng ban khác trong tổ chức. Trưởng ban Bộ phận KTNB phải được báo cáo công việc trực tiếp và được bổ nhiệm bởi Ban kiểm soát nhà trường để thể hiện được sự khách quan khi thực hiện nhiệm vụ. Biến $\mathrm{OB}$ dựa trên nghiên cứu của Cohen et al., (2004); Mat Zain và Subramaniam (2007); IIA, (2017).

- SIA: là biến đại diện phạm vi công việc của Bộ phận KTNB khi tổ chức Bộ phận này. Biến SIA thể hiện phạm vi kiểm toán mở rộng của KTNB để thể hiện vai trò cần thiết của việc tổ chức Bộ phận này. Mặt khác việc xác định phạm vi hoạt động của Bộ phận KTNB cũng phụ thuộc vào các kỹ năng nghề nghiệp thông qua sự hiểu biết của nhân viên kiểm toán về hệ thống công nghệ thông tin được trang bị hỗ trợ cho hoạt động kiểm toán. Biến này cũng chịu ảnh hưởng bởi cách thức tổ chức hệ thống giấy tờ làm việc chuẩn hóa phù hợp với công tác kiểm toán. Biến SIA dựa trên nghiên cứu của Al-Twaijry et al, (2003); Fadzil et al., (2005).

Từ đó, mô hình nghiên cứu như sau:

$$
\mathbf{I A R}=\beta_{0}+\beta_{1} \mathbf{I M}+\beta_{2} \mathbf{I S}+\beta_{3} \mathbf{S M}+\beta_{4} \mathbf{I A}+\beta_{5} \mathbf{O B}+\beta_{6} \mathbf{S I A}+\boldsymbol{\epsilon}_{\mathbf{t}}
$$




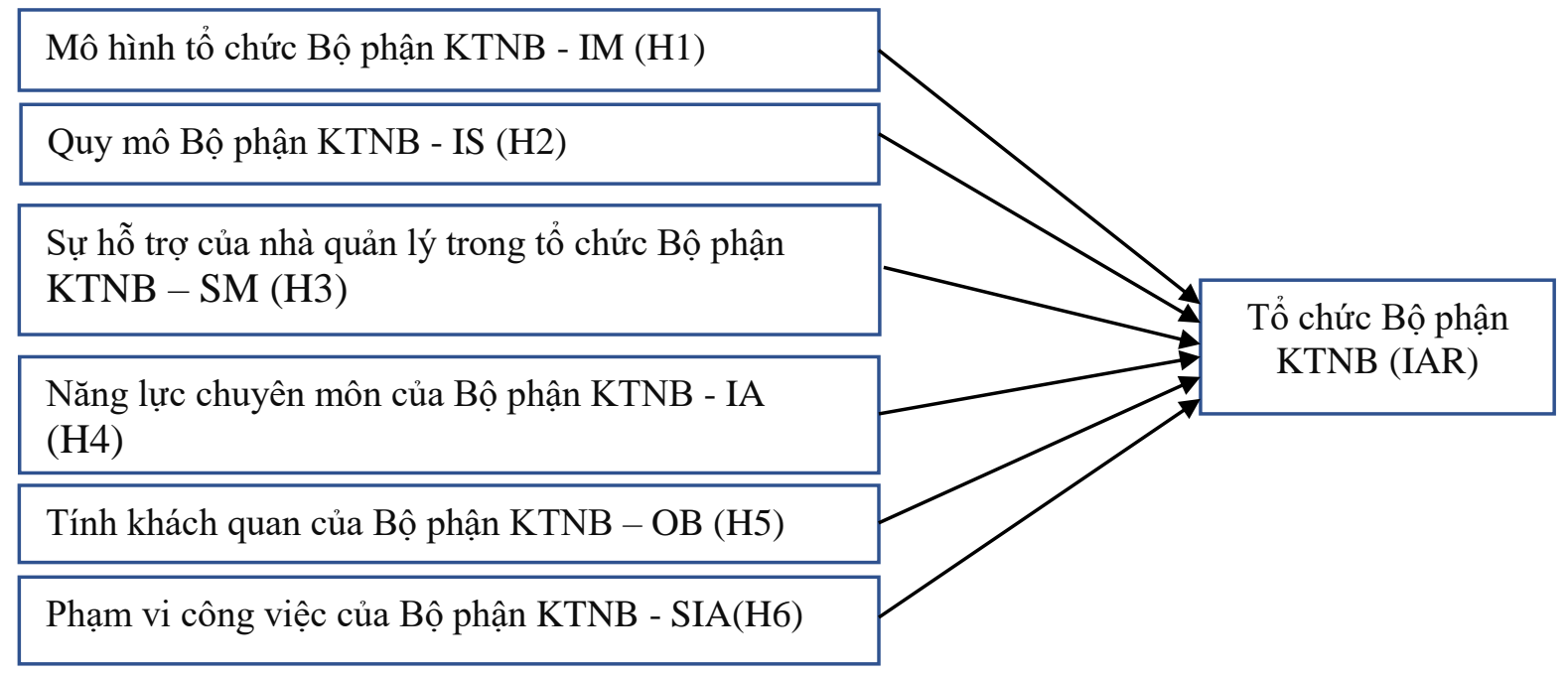

Hình 1: Mô hình nghiên cứu

Trên cơ sở mô hình nghiên cứu, các giả thuyết nghiên cứu bao gồm:

Giả thuyết H1: Mô hình tổ chức Bộ phận KTNB có ảnh hưởng đến việc tổ chức Bộ phận KTNB tại các trường Đại học công lập theo cơ chế tự chủ Việt Nam.

Giả thuyết H2: Quy mô Bộ phận KTNB có ảnh hưởng đến tổ chức Bộ phận KTNB tại các trường Đại học công lập theo cơ chế tự chủ Việt Nam.

Giả thuyết H3: Sự hỗ trợ của Ban quản lý trong tổ chức Bộ phận KTNB có ảnh hưởng đến tổ chức Bộ phận KTNB tại các trường Đại học công lập theo cơ chế tự chủ Việt Nam.

Giả thuyết H4: Năng lực chuyên môn của Bộ phận KTNB có ảnh hưởng đến tổ chức Bộ phận KTNB tại các trường Đại học công lập theo cơ chế tự chủ Việt Nam.

Giả thuyết H5: Tính khách quan của Bộ phận KTNB có ảnh hưởng đến tổ chức Bộ phận KTNB tại các trường Đại học công lập theo cơ chế tự chủ Việt Nam.

Giả thuyết H6: Phạm vi công việc của Bộ phận KTNB có ảnh hưởng đến tổ chức Bộ phận KTNB tại các trường các trường Đại học công lập theo cơ chế tự chủ Việt Nam.

Dựa trên mô hình nghiên cứu và các giả thuyết nghiên cứu, chúng tôi tiến hành hoàn thiện bộ thang đo cho các yếu tố trong mô hình, chi tiết theo Bảng 1 .

Bảng 1: Tổng hợp thang đo các biến trong mô hình

\begin{tabular}{|c|c|c|}
\hline Nhân tố & Mã hóa & Biến quan sát \\
\hline \multirow{3}{*}{$\begin{array}{l}\text { Mô hình tổ chức } \\
\text { Bộ phận KTNB } \\
\text { (IM) }\end{array}$} & IM1 & Bộ phận KTNB trực thuộc Ban giám hiệu nhà trường \\
\hline & IM2 & Bộ phận KTNB trực thuộc Ban kiểm soát nhà trường \\
\hline & IM3 & Tỷ lệ số lượng KTV nội bộ trên số lượng nhân viên đơn vị \\
\hline \multirow{3}{*}{$\begin{array}{l}\text { Quy mô của Bộ } \\
\text { phận KTNB (IS) }\end{array}$} & IS1 & Quy mô và loại hình hoạt động của đơn vị \\
\hline & IS2 & Nhiệm vụ chức năng của Bộ phận KTNB \\
\hline & IS3 & Quy mô nhóm kiểm toán \\
\hline \multirow{3}{*}{$\begin{array}{l}\text { Sự hỗ trợ của nhà } \\
\text { quản lý trong tổ } \\
\text { chức Bộ phận } \\
\text { KTNB }(\mathrm{SM})\end{array}$} & SM1 & Có chính sách đào tạo hàng năm dành cho nhân viên KTNB \\
\hline & SM2 & Ngân sách đầu tư hàng năm cho Bộ phận KTNB \\
\hline & SM3 & Thực hiện chế độ hậu đãi cho nhân viên Bộ phận KTNB \\
\hline \multirow{3}{*}{$\begin{array}{l}\text { Năng lực chuyên } \\
\text { môn của Bộ phận } \\
\text { KTNB (IA) }\end{array}$} & IA1 & Kinh nghiệm của nhân viên Bộ phận KTNB \\
\hline & IA2 & Bằng cấp chuyên môn của nhân viên Bộ phận KTNB \\
\hline & IA3 & Chương trình cập nhật kiến thức hàng năm dành cho KTNB \\
\hline
\end{tabular}




\begin{tabular}{|c|c|c|}
\hline \multirow{3}{*}{$\begin{array}{l}\text { Tính khách quan } \\
\text { của Bộ phận KTNB } \\
\text { (OB) }\end{array}$} & OB1 & Tính độc lập Bộ phận KTNB so với bộ phận khác \\
\hline & OB2 & $\begin{array}{l}\text { Trưởng ban Bộ phận KTNB báo cáo công việc trực tiếp cho Ban } \\
\text { kiềm soát nhà trường }\end{array}$ \\
\hline & OB3 & Trưởng Bộ phận KTNB được bổ nhiệm bởi Ban kiểm soát nhà trường \\
\hline \multirow{3}{*}{$\begin{array}{l}\text { Phạm vi công việc } \\
\text { của Bộ phận KTNB } \\
\text { (SIA) }\end{array}$} & SIA 1 & Phạm vi kiểm toán của Bộ phận KTNB \\
\hline & SIA 2 & Kỹ năng nghề nghiệp của nhân viên Bộ phận KTNB \\
\hline & SIA 3 & Hệ thống giấy tờ làm việc của kiểm toán \\
\hline \multirow{3}{*}{$\begin{array}{l}\text { Tổ chức Bộ phận } \\
\text { KTNB (IAR) }\end{array}$} & IAR1 & Áp lực từ quy định pháp lý \\
\hline & IAR2 & Áp lực nâng cao hiệu quả kiểm soát hoạt động tổ chức \\
\hline & IAR3 & Yêu cầu của Ban kiểm soát nhà trường \\
\hline
\end{tabular}

\subsection{Phương pháp nghiên cứu}

Nghiên cứu được chúng tôi thực hiện qua các giai đoạn như sau:

Giai đoạn 1: Dựa vào tổng quan các nghiên cứu trước, chúng tôi sử dụng phương pháp định tính thảo luận với chuyên gia nhằm khám phá thêm các nhân tố mới và xây dựng mô hình nghiên cứu chính thức.

Giai đoạn 2: Dựa trên kết quả định tính, chúng tôi tiến hành thiết kế bảng câu hỏi và khảo sát các đối tượng có liên quan. Kết quả khảo sát được nhập liệu và kiểm định bằng hệ số Crombach's Alpha.

Giai đoạn 3: Chúng tôi tiến hành phân tích nhân tố khám phá EFA và phân tích hồi quy tuyến tính đa biến để kiểm định các giả thuyết của mô hình. Từ đó xem xét mức độ ảnh hưởng của các nhân tố này đến việc tổ chức Bộ phận KTNB tại các trường Đại học công lập theo cơ chế tự chủ Việt Nam.

\section{KẾT QUẢ NGHIÊN CÚU}

\subsection{Thống kê mẫu nghiên cứu}

Đối tượng tham gia phỏng vấn trực tiếp và phỏng vấn qua thư điện tử trong nghiên cứu định tính là các kiểm toán viên (KTV) nội bộ, kiểm soát viên nội bộ. Sau đó, từ thanh đo đã được xây dựng chính thức, chúng tôi tiến hành khảo sát để thu thập dữ liệu nghiên cứu định lượng. Với thang đo bao gồm 18 nhân tố, cho nên kích thước mẫu tối thiểu là $18 \times 5=90$ (Hair ct al., 2010).

Do đối tượng nghiên cứu là các trường Đại học công lập theo cơ chế tự chủ nói chung và trường Đại học Công nghiệp TPHCM nói riêng chưa có hoạt động KTNB. Cho nên, để đạt được tối thiểu 90 quan sát, chúng tôi tiến hành gửi bảng câu hỏi khảo sát trực tiếp hoặc thông qua hộp thư điện tử đến 250 cán bộ nhân viên làm việc tại các phòng ban gần với chức năng KTNB trong 05 trường Đại học gồm có: trường Đại học Công nghiệp TP.HCM, trường Đại học Kinh Tế - Luật, trường Đại học Tài chính Marketing, trường Đại học Mở, trường Đại học Tôn Đức Thắng, các nhà nghiên cứu và chuyên gia kế toán kiểm toán.

Sau 05 tháng (tháng 10/2017 - 02/2018) kể từ ngày gửi bảng câu hỏi, chúng tôi đã thu hồi được 153 bảng trả lời khảo sát hợp lệ, đạt tỷ lệ khoảng 62\% (153/250). Dữ liệu từ 153 bảng trả lời này được chúng tôi nhập vào phần mềm SPSS để sử dụng chính thức cho các phân tích tiếp theo của nghiên cứu.

Bảng 2. Thống kê số lượng phản hồi khảo sát

\begin{tabular}{|l|c|c|c|}
\hline \multicolumn{1}{|c|}{ Đối tượng khảo sát } & \multirow{2}{*}{$\begin{array}{c}\text { Số lượng gửi bảng } \\
\text { khảo sát }\end{array}$} & \multicolumn{2}{c|}{$\begin{array}{c}\text { Số lượng phản hồi khảo } \\
\text { sát }\end{array}$} \\
\cline { 3 - 4 } & & Tổng số & Hợp lệ \\
\hline Nhân viên trong các trường Đại học & 110 & 90 & 73 \\
\hline Nhà nghiên cứu trong lĩnh vực kế toán kiểm toán & 60 & 50 & 40 \\
\hline KTV nội bộ, kiểm soát viên, kế toán viên & 50 & 35 & 35 \\
\hline Kiểm toán viên độc lập, kiềm toán viên nhà nước & 30 & 16 & 5 \\
\hline Tổng cộng & $\mathbf{2 5 0}$ & $\mathbf{1 9 1}$ & $\mathbf{1 5 3}$ \\
\hline
\end{tabular}




\subsection{Kết quả kiểm định thang đo}

Kết quả kiểm định thang đo (chi tiết theo Bảng 3) cho thấy thang đo có độ chính xác khá cao khi 17 biến quan sát đều có hệ số Crombach Alpha > 0.7, và một biến quan sát IS2 có hệ số Crombach Alpha> 0.6. Kết quả này đã chứng minh thang đo vẫn đảm bảo độ tin cậy cần thiết vì khá nhiều nhà nghiên cứu đề nghị rằng Crombach Alpha từ 0.6 trở lên là có thể sử dụng được trong điều kiện nhân tố đang nghiên cứu là nhân tố mới đối với người trả lời trong bối cảnh nghiên cứu (Hoàng Trọng \& Chu Nguyễn Mộng Ngọc, 2008; Nguyễn Đình Thọ, 2011). Đồng thời tất cả các biến quan sát đều có hệ số tương quan biến tổng điều chỉnh trong từng thang đo thành phần lớn hơn 0.3 , do đó các thanh đo này đều được chấp nhận sử dụng chính thức cho nghiên cứu.

Bảng 3. Kết quả kiểm định chính thức độ tin cậy của thang đo

\begin{tabular}{|c|c|c|c|c|}
\hline Thang đo & $\begin{array}{c}\text { Trung bình thang đo } \\
\text { nếu loại biến tổng }\end{array}$ & $\begin{array}{c}\text { Phương sai thang đo } \\
\text { nếu loại biến tổng }\end{array}$ & Tương quan biến tổng & $\begin{array}{l}\text { Cronbach's Alpha nếu } \\
\text { loại biến tổng }\end{array}$ \\
\hline \multicolumn{4}{|c|}{ Nhân tố Mô hình tồ chức Bộ phận KTNB } & 0.854 \\
\hline IM1 & 7.69 & 2.533 & .722 & .803 \\
\hline IM2 & 7.14 & 2.619 & .770 & .755 \\
\hline IM3 & 7.11 & 2.836 & .691 & .829 \\
\hline \multicolumn{4}{|c|}{ Nhân tố Quy mô Bộ phận KTNB } & $\mathbf{0 . 8 1 3}$ \\
\hline IS1 & 8.00 & 2.145 & .609 & .799 \\
\hline IS2 & 8.01 & 1.947 & .726 & .676 \\
\hline IS5 & 7.96 & 2.143 & .658 & .748 \\
\hline \multicolumn{4}{|c|}{ Nhân tố Sự hỗ trợ của nhà quản lý trong tổ chức Bộ phận KTNB } & $\mathbf{0 . 8 5 2}$ \\
\hline SM1 & 7.51 & 2.252 & .730 & .788 \\
\hline SM2 & 7.77 & 2.467 & .682 & .833 \\
\hline SM3 & 7.57 & 1.944 & .770 & .751 \\
\hline \multicolumn{4}{|c|}{ Nhân tố Năng lực chuyên môn của Bộ phận KTNB } & $\mathbf{0 . 8 1 1}$ \\
\hline IA1 & 7.90 & 1.634 & .588 & .819 \\
\hline IA2 & 7.94 & 1.529 & .695 & .704 \\
\hline IA3 & 7.86 & 1.672 & .708 & .698 \\
\hline \multicolumn{4}{|c|}{ Nhân tố Tính khách quan của Bộ phận KTNB } & $\mathbf{0 . 8 3 8}$ \\
\hline OB1 & 7.54 & 2.487 & .663 & .814 \\
\hline OB2 & 7.65 & 2.557 & .734 & .747 \\
\hline OB3 & 7.59 & 2.414 & .710 & .766 \\
\hline \multicolumn{4}{|c|}{ Nhân tố Phạm vi công việc của Bộ phận KTNB } & 0.794 \\
\hline SIA1 & 8.47 & 1.567 & .627 & .731 \\
\hline SIA2 & 8.49 & 1.541 & .644 & .715 \\
\hline SIA3 & 8.50 & 1.304 & .650 & .713 \\
\hline
\end{tabular}

\subsection{Phân tích nhân tố khám phá EFA}

Kết quả phân tích ở Bảng 4 cho thấy hệ số $\mathrm{KMO}=0.822>0.7$, điều này có nghĩa là dữ liệu nghiên cứu thu thập phù hợp với phân tích nhân tố khám phá (EFA). Kiểm định Bartlett Test có giá trị Sig. $=$ $0.000<0.05$ cho thấy các biến quan sát có tương quan với nhau trong mỗi nhóm nhân tố.

Bảng 4. Kết quả kiểm định $\mathrm{KMO}$ và Bartlett Test $\mathrm{KMO}$ và Bartlett's Test

\begin{tabular}{|l|l|r|}
\hline \multicolumn{2}{|l|}{ Kaiser-Meyer-Olkin Measure of Sampling Adequacy. } & .822 \\
\hline \multirow{3}{*}{ Bartlett's Test of Sphericity } & Approx. Chi-Square & 1352.016 \\
\cline { 2 - 3 } & df & 153 \\
\cline { 2 - 3 } & Sig. & .000 \\
\hline
\end{tabular}


Đồng thời, kết quả phân tích nhân tố khám phá ở Bảng 5 cho thấy tại giá trị Eigenvalue $>1$ có tất cả 6 nhân tố được trích ra và tổng phương sai trích cộng dồn Cumulative $\%=75.922$ lớn hơn $50 \%$. Kết quả này cho biết $75.922 \%$ biến thiên của nhân tố nghiên cứu được giải thích bởi các biến quan sát thành phần. Các thuộc tính đều đạt yêu cầu nên thang đo có giá trị cao.

Bảng 5. Kết quả phân tích nhân tố khám phá

\begin{tabular}{|c|c|c|c|c|c|c|c|c|c|}
\hline \multirow[t]{2}{*}{$\begin{array}{c}\text { Nhân } \\
\text { tố }\end{array}$} & \multicolumn{3}{|c|}{ Initial Eigenvalues } & \multicolumn{3}{|c|}{$\begin{array}{c}\text { Extraction Sums of Squared } \\
\text { Loadings }\end{array}$} & \multicolumn{3}{|c|}{$\begin{array}{c}\text { Rotation Sums of Squared } \\
\text { Loadings } \\
\end{array}$} \\
\hline & Total & $\begin{array}{c}\% \text { of } \\
\text { Variance }\end{array}$ & $\begin{array}{c}\text { Cumulative } \\
\%\end{array}$ & Total & $\begin{array}{c}\% \text { of } \\
\text { Variance }\end{array}$ & $\begin{array}{c}\text { Cumulative } \\
\%\end{array}$ & Total & $\begin{array}{c}\% \text { of } \\
\text { Variance }\end{array}$ & $\begin{array}{c}\text { Cumulative } \\
\%\end{array}$ \\
\hline 1 & 5.438 & 30.210 & 30.210 & 5.438 & 30.210 & 30.210 & 2.411 & 13.397 & 13.397 \\
\hline 2 & 3.299 & 18.330 & 48.540 & 3.299 & 18.330 & 48.540 & 2.360 & 13.109 & 26.506 \\
\hline 3 & 1.700 & 9.443 & 57.982 & 1.700 & 9.443 & 57.982 & 2.263 & 12.570 & 39.076 \\
\hline 4 & 1.155 & 6.416 & 64.399 & 1.155 & 6.416 & 64.399 & 2.226 & 12.364 & 51.440 \\
\hline 5 & 1.069 & 5.938 & 70.337 & 1.069 & 5.938 & 70.337 & 2.220 & 12.333 & 63.773 \\
\hline 6 & 1.005 & 5.585 & 75.922 & 1.005 & 5.585 & 75.922 & 2.187 & 12.150 & 75.922 \\
\hline
\end{tabular}

Bảng 6. Bảng phân tích nhân tố

\begin{tabular}{|c|c|c|c|c|c|c|}
\hline & & & Nhâ & & & \\
\hline & 1 & 2 & 3 & 4 & 5 & 6 \\
\hline SM2 & .827 & & & & & \\
\hline SM1 & .820 & & & & & \\
\hline SM3 & .816 & & & & & \\
\hline OB3 & & .859 & & & & \\
\hline OB2 & & .857 & & & & \\
\hline OB1 & & .801 & & & & \\
\hline IM3 & & & .857 & & & \\
\hline IM2 & & & .778 & & & \\
\hline IM1 & & & 687 & & & \\
\hline SIA3 & & & & .820 & & \\
\hline SIA1 & & & & .803 & & \\
\hline SIA2 & & & & .801 & & \\
\hline IA3 & & & & & .832 & \\
\hline IA2 & & & & & .773 & \\
\hline IA1 & & & & & .714 & \\
\hline IS2 & & & & & & .880 \\
\hline IS1 & & & & & & .816 \\
\hline IS3 & & & & & & .783 \\
\hline
\end{tabular}

\subsection{Phân tích tương quan của các nhân tố}

Phân tích tương quan Pearson cũng được chúng tôi sử dụng để xem xét mối quan hệ tương quan giữa biến phụ thuộc và biến độc lập. Kết quả phân tích cho thấy mức ý nghĩa Sig. của môi tương quan giữa nhân tố Quy mô Bộ phận KTNB (IS) và biến phụ thuộc (IAR) là $0.296>0.05$ cho nên nhân tổ này không được đưa vào mô hình để phân tích hồi quy. Giá trị Sig. của 5 nhân tố còn lại đều $<0.05$ chứng tỏ các biến này đều có ý nghĩa thống kê hay nói cách khác các biến trong mô hình đều phù hợp đưa vào mô hình nghiên cứu. Mặt khác, hệ số tương quan tuyệt đối giữa các biến từ 0.184 đến $0.713<=$ hệ số điều kiện 0.8 , giá trị phân biệt đã đạt được, các thang đo này đã đo lường được các khái niệm nghiên cứu khác nhau. 


\subsection{Phân tích hồi quy tuyến tính đa biến}

Phân tích hồi quy đa biến là nghiên cứu mức độ ảnh hưởng của nhiều biến độc lập đến một biến phụ thuộc. Để thực hiện đo lường ảnh hưởng của các nhân tố đến tổ chức Bộ phận KTNB tại các trường Đại học công lập theo cơ chế tự chủ Việt Nam, chúng tôi tiến hành phân tích hồi quy đa biến và kiểm định các giả thuyết với 5 nhóm nhân tố là biến độc lập. Kết quả nghiên cứu cho thấy mức độ ảnh hưởng của các nhân tố được thể hiện qua hệ số hồi quy đã chuẩn hóa vì hệ số này không phụ thuộc thang đo (Nguyễn Dình Thọ, 2011). Do đó, phương trình hồi quy đa biến đã chuẩn hóa để phân tích mức độ ảnh hưởng của các nhân tố đến tổ chức Bộ phận KTNB tại các trường Đại học công lập theo cơ chế tự chủ Việt Nam.

Kết quả Bảng 7 cho thấy hệ số tương quan hiệu chỉnh $\mathrm{R}^{2}=0.593$, có nghĩa là $59,3 \%$ sự thay đổi của biến phụ thuộc được giải thích bởi biến độc lập. Hệ số Durbin Watson $(\mathrm{d})=2.100<3$ chứng tỏ mô hình không có hiện tượng tự tương quan phần dư. Điều này cũng cho thấy mô hình nghiên cứu phù hợp.

Bảng 7. Bảng tóm tắt mô hình

\begin{tabular}{|l|r|r|r|r|r|}
\hline Mô hình & $\begin{array}{c}\text { Hệ số } \\
\mathrm{R}\end{array}$ & $\begin{array}{c}\text { Hệ số xác định } \\
\mathrm{R}^{2}\end{array}$ & $\begin{array}{c}\text { Hệ số xác định hiệu } \\
\text { chỉnh } \mathrm{R}^{2}\end{array}$ & $\begin{array}{c}\text { Sai số chuẩn } \\
\text { ước tính }\end{array}$ & $\begin{array}{c}\text { Hệ số } \\
\text { Durbin-Watson }\end{array}$ \\
\hline 1 & $.779^{\mathrm{a}}$ & .606 & .593 & .41235 & 2.108 \\
\hline
\end{tabular}

Bảng 8 cho thấy kiểm định trị thống kê $\mathrm{F}=45.295$ với giá trị Sig. $=0.000$, chứng tỏ rằng mô hình hồi quy xây dựng là phù hợp với nguồn dữ liệu thu thập được và các biến đưa vào mô hình nghiên cứu đều có ý nghĩa thống kê với mức ý nghĩa 5\%, các biến độc lập trong mô hình có quan hệ với biến phụ thuộc.

Bảng 8. Phân tích ANOVA - Độ tin cậy của mô hình nghiên cứu

\begin{tabular}{|l|l|r|r|r|r|r|}
\hline \multicolumn{2}{|c|}{ Mô hình } & $\begin{array}{r}\text { Tổng độ lệch bình } \\
\text { phương }\end{array}$ & $\begin{array}{c}\text { Bậc tự do } \\
\text { Df }\end{array}$ & Phương sai & $\begin{array}{c}\text { Kiểm định } \\
\text { F }\end{array}$ & $\begin{array}{c}\text { Mức ý nghĩa } \\
\text { Sig. }\end{array}$ \\
\hline \multirow{3}{*}{1} & Hồi quy & 38.509 & 5 & 7.702 & 45.295 & $.000^{b}$ \\
& Phần dư & 24.995 & 147 & .170 & & \\
& Tổng & 63.503 & 152 & & & \\
\hline
\end{tabular}

Bảng 9 cho thấy mô hình nghiên cứu có 1 biến phụ thuộc và 5 biến độc lập được phân tích hồi quy bằng phương pháp Enter, loại biến độc lập Tính khách quan của Bộ phận KTNB $(\mathrm{OB})$ vì có giá trị Sig. lớn hơn 0.05 . Hệ số VIF của các biến độc lập đều $<2$ cho thấy không có hiện tượng đa cộng tuyến tức là không có mối quan hệ tuyến tính nào giữa các biến.

Bảng 9. Kết quả phân tích mô hình hồi quy đa biến

\begin{tabular}{|c|c|c|c|c|c|c|c|}
\hline \multirow[t]{2}{*}{ Mô hình } & \multicolumn{2}{|c|}{$\begin{array}{c}\text { Hệ số chưa chuẩn } \\
\text { hóa }\end{array}$} & \multirow{2}{*}{$\begin{array}{c}\begin{array}{c}\text { Hệ số chuẩn } \\
\text { hóa }\end{array} \\
\text { Beta }\end{array}$} & \multirow[t]{2}{*}{ Giá trị t } & \multirow[t]{2}{*}{$\begin{array}{c}\text { Mức ý } \\
\text { nghĩa }\end{array}$} & \multicolumn{2}{|c|}{ Thống kê cộng tuyến } \\
\hline & $\begin{array}{l}\text { Trọng số } \\
\text { hồi quy }\end{array}$ & $\begin{array}{l}\text { Sai lệch } \\
\text { chuẩn }\end{array}$ & & & & $\begin{array}{c}\text { Hệ số chấp } \\
\text { nhận }\end{array}$ & $\begin{array}{c}\text { Hệ số } \\
\text { phóng đại } \\
\text { phuông sai } \\
\text { VIF }\end{array}$ \\
\hline (Constant) & .123 & .337 & & .363 & .717 & & \\
\hline SM & .181 & .059 & .201 & 3.040 & .003 & .613 & 1.631 \\
\hline IM & .369 & .057 & .450 & 6.443 & .000 & .549 & 1.823 \\
\hline IA & .239 & .072 & .224 & 3.320 & .001 & .587 & 1.702 \\
\hline OB & .018 & .050 & .021 & .362 & .718 & .790 & 1.266 \\
\hline SIA & .156 & .060 & .139 & 2.592 & .011 & .927 & 1.079 \\
\hline
\end{tabular}


Như vậy, mô hình hồi quy phù hợp với dữ liệu khảo sát là:

$$
\mathrm{IAR}=\mathbf{0 . 4 5 0 I M}+\mathbf{0 . 2 2 4} \mathrm{IA}+\mathbf{0 . 2 0 1 S M}+\mathbf{0 . 1 3 9 S I A}+\boldsymbol{\epsilon}_{\mathrm{t}}
$$

Kết quả này chính là cơ sở để đề xuất các kiến nghị nhằm nâng cao hiệu quả tổ chức Bộ phận KTNB tại đơn vị.

\section{KẾT LUẬN}

Từ kết quả nghiên cứu cho thấy có 4 nhân tố ảnh hưởng đến tổ chức Bộ phận KTNB tại các trường Đại học công lập Việt Nam nói chung và trường Đại học Công nghiệp TPHCM nói riêng. Các nhân tố này có mức độ ảnh hưởng theo thứ tự là: Mô hình tổ chức Bộ phận KTNB, (2) Năng lực chuyên môn của Bộ phận KTNB, (3) Sự hỗ trợ của Ban quản lý trong tổ chức Bộ phận KTNB, (4) Phạm vi công việc của Bộ phận KTNB. Trên cơ sở tham khảo tình hình thực tế tại đơn vị và kết quả điều tra khảo sát, chúng tôi đề xuất một số giải pháp như sau:

- Mô hình tổ chức Bộ phận KTNB thường phụ thuộc vào đặc điểm quản lý của đơn vị. Do trường Đại học Công nghiệp TP.HCM là trường Đại học công lập, không có Hội đồng quản trị và Ban kiểm soát như các tổ chức kinh doanh khác, người quản lý cao nhất là Ban giám hiệu, quy mô trường lớn và đa dạng trong hoạt động, vì thế mô hình tổ chức Bộ phận KTNB hiện nay do Ban giám hiệu nhà trường quản lý là thích hợp, tương lai vai trò này nên giao cho Hội đồng trường. Đồng thời, để đảm bảo tính độc lập của Bộ phận KTNB, việc bổ nhiệm, miễn nhiệm và trách nhiệm báo cáo của trưởng Bộ phận KTNB nên do Ban giám hiệu nhà trường hay Hội đồng trường quyết định. Về quy mô Bộ phận KTNB, số lượng nhân viên trong Bộ phận này có thể được xác định dựa vào các yếu tố khách quan và chủ quan của nhà trường nhu: số lượng nhân viên của toàn trường, tính đa dạng của lĩnh vực kinh doanh và khu vực địa lý hoạt động. Dựa theo nghiên cứu của Nguyễn Phú Giang (2015), cứ trung bình 1.000 nhân viên trong nhà trường thì Bộ phận KTNB cần từ 01 đến 04 kiểm toán viên. Mặt khác, trường Đại học Công nghiệp TP.HCM có ngành nghề kinh doanh đa dạng bao gồm giáo dục đào tạo, nhà xuất bản, sản xuất nước đóng chai,..., cho nên số lượng kiểm toán viên nội bộ càng cần nhiều hơn. Trường Đại học Công nghiệp TP.HCM có 01 cơ sở chính tại TP.HCM, 02 cơ sở tại Quảng Ngãi và Thanh Hóa, với khu vực hoạt động phân tán như vậy, số lượng kiểm toán viên nội bộ cũng cần nhiều thêm. Kết quả nghiên cứu của Powell (1993); Dirsmith et al., (2000) cũng cho thấy Bộ phận KTNB nên được chia thành nhiều nhóm kiểm toán với mối nhóm có từ 3 đến 5 nhân viên để thực hiện hiệu quả công tác kiểm toán được giao.

- Về năng lực chuyên môn của Bộ phận KTNB: Tổ chức Bộ phận KTNB cũng cần chú ý đến việc xây dựng một cơ cấu nguồn nhân sự phù hợp, đáp ứng được mục tiêu đề ra. Do đó, cần có quy chế rõ ràng, cụ thể đảm bảo yêu cầu về trình độ, năng lực chuyên môn ngay từ khâu tuyển dụng. Cần tuyển dụng những nhân viên, cán bộ là những người đã tốt nghiệp Đại học chuyên ngành; có thời gian kinh nghiệm ít nhấn là 5 năm trở lên và phải có tối thiểu 3 năm làm việc trong lĩnh vực kiểm toán. Nếu nhà trường sử dụng nguồn nhân sự sẵn có cho Bộ phận KTNB thì cần quan tâm đến kỉnh nghiệm công tác, sự hiểu biết về lĩnh vực công tác và kiến thức chuyên môn về kiểm soát nội bộ, kế toán tài chính, kiểm toán...Trước mắt, họ có thể tham gia các khóa học đào tạo ngắn hạn nhưng về lâu dài, nhà trường cần xây dựng một chương trình đào tạo chuyên sâu hàng năm dành cho nhân viên Bộ phận KTNB. Đối với các nhân viên KTNB đã đáp ứng được yêu cầu đào tạo và kinh nghiệm kiểm toán thì vẫn phải tham gia cập nhật thường xuyên các kiến thức nghiệp vụ chuyên môn trong nước và quốc tế. Đồng thời, nhà trường cũng cần xây dựng chương trình đánh giá rủi ro hoạt động để Bộ phận KTNB nhận diện đúng đắn được vấn đề xảy ra và mức độ ảnh hưởng. Từ đó, có thể xây dựng được các thủ tục kiểm soát hữu hiệu cho nhà trường, chứng minh được năng lực chuyên môn của Bộ phận KTNB.

- Về sự hỗ trợ của Ban quản lý nhà trường trong tổ chức Bộ phận KTNB: Sự ủng hộ, quan tâm và hỗ trợ cần thiết từ Ban quản lý nhà trường là điều hết sức cần thiết để tổ chức thành công Bộ phận KTNB. Theo đó, nhà trường cần có các chính sách đào tạo hàng năm dành cho nhân viên trong Bộ phận KTNB bằng cách tổ chức các khóa học chuyên môn hoặc gửi nhân viên đến tham gia các chương trình đào tạo được tổ chức bởi Bộ tài chính hoặc các Hiệp hội nghề nghiệp quốc tế tại Việt Nam. Nhà trường cũng cần xây dựng các chính sách đãi ngộ lương, thưởng và phụ cấp trách nhiệm tương ứng với trình độ và cống hiến của KTV nội bộ. Việc lập dự toán nguồn ngân sách hoạt động cho Bộ phận KTNB cũng cần được 
thực hiện ngay từ đầu mỗi năm tài chính và công khai để Bộ phận KTNB có thể lập các kế hoạch kiểm toán, phân công đội ngũ kiểm toán viên và thực hiện tốt công tác kiểm toán.

- Về phạm vi công việc KTNB: Đối với nhà quản lý, phạm vi kiểm toán chính là nhân tố làm tăng giá trị và sự cần thiết của Bộ phận KTNB. Do đó, Bộ phận này cần thực hiện kết hợp ba phạm vi kiểm toán hoạt động, kiểm toán tuân thủ và kiểm toán BCTC thông qua đánh giá chiến lược hoạt động và hệ thống KSNB trong nhà trường, đánh giá sự trung thực, hợp lý của thông tin trọng yếu trên Báo cáo tài chính và đánh giá việc tuân thủ pháp luật quy định của nhà trường. Để đảm bảo tính hiệu quả khi thực hiện mở rộng phạm vi kiểm toán, Bộ phận KTNB cần được hỗ trợ bởi các kỹ năng nghề nghiệp tốt của nhân viên kiểm toán, phần mềm kiểm toán được xây dựng hoàn thiện cùng với hệ thống giấy tờ làm việc được tổ chức đầy đủ tương thích với mọi đối tượng kiểm toán.

- Về phía nhà nước, cần nhanh chóng ban hành Hệ thống chuẩn mực hướng dẫn hoạt động KTNB để tạo cơ sở thống nhất cho hoạt động này tại Việt Nam. Bước đầu, Bộ Tài Chính có thể tham khảo Hệ thống chuẩn mực KTNB quốc tế. Sau đó, Bộ Tài Chính có thể tham khảo ý kiến đóng góp của các công ty kiểm toán để điều chỉnh cho phù hợp với bối cảnh Việt Nam. Bộ Tài Chính cũng nên xem xét việc thành lập Hiệp hội nghề nghiệp KTNB để giao nhiệm và soạn thảo, xây dựng Hệ thống chuẩn mực KTNB Việt Nam. Các kỳ thi chuyên môn và cẩp chứng chỉ hành nghề KTNB nên được thực hiện để tạo cơ sở pháp lý vững chắc cho nghề nghiệp này tại Việt Nam. Đồng thời khung pháp lý về quản lý hoạt động KSNB cũng nên được ban hành để hỗ trợ cho việc kiểm soát chất lượng hoạt động KTNB trong tương lai. Như vậy, Bộ phận KTNB có thể hỗ trợ nhà trường phát hiện những yếu kém, gian lận trong quản lý, bảo vệ tài sản của nhà trường cũng là bảo vệ tài sản của nhà nước; đề xuất các giải pháp nhằm cải tiến, hoàn thiện hệ thống quản lý, điều hành hoạt động của nhà trường.

Đề tài nghiên cứu tập trung vào các nhân tố ảnh hưởng đến tổ chức Bộ phận KTNB tại các trường Đại học công lập theo cơ chế tự chủ và trường Đại học Công nghiệp TP.HCM. Tuy nhiên, vì đây là đề tài mới nên chưa thực hiện điều tra được một số nhân tố khác có ảnh hưởng đến việc tổ chức Bộ phận KTNB như: ảnh hưởng của các bên liên quan, văn hóa tổ chức, năng lực chuyên môn và tính khách quan của Ban quản lý nhà trường. Mặt khác, đề tài cũng chưa nghiên cứu đến quy trình tổ chức và xây dựng Bộ phận KTNB. Đây chính là những hướng nghiên cứu tiếp theo trong tương lai để nâng cao hiệu quả tổ chức Bộ phận KTNB tại các trường Đại học công lập Việt Nam nói chung và trường Đại học Công nghiệp TP.HCM nói riêng.

\section{TÀI LIỆU THAM KHẢO}

1. Adam Smith, (1776). The Wealth of Nations E. Cannan (Ed.). Retrieved from http://site.ebrary.com.ezproxy.ecu.edu.au/lib/ecu/docDetail.action?docID=10381 174

2. Akerlof G.A, (1970). The Market for "Lemons": Quality Uncertainty and the Market Mechanism. Quarterly Journal of Economics.

3. Albrecht, W. S., Howe, K. R., Schueler, D. R. \& Stocks, K. D., (1988). Evaluating the Effectiveness of Internal Audit Departments (Altamonte Spring, FL: Institute of Internal Auditors).

4. Allegrini, M., D’Onza, G., Melville, R., Paape, L. \& Sarens, G., (2006). The European literature review on internal auditing. Managerial Auditing Journal. Vol. 21, No. 8.

5. Anderson Urton L. et al, (2010). Effective Sizing of Internal Audit Departments, Tehe Institute of Internal Auditors Research Foundation.

6. Al-Twaijry, A.Brierley \& Gwilliam D, (2003). The development of internal audit in Saudi Arabia: An institutional theory perspective. Critical Perspectives on Accounting, 14, 507-531.

7. Arena, M., \& Azzone G., (2009). Identifying organizational drivers of internal audit effectiveness, International Journal of Auditing, 13, 43-60. 
8. Ashton, A. H., (1991). Experience and error frequency knowledge as potential determinants of audit expertise. The Accounting Review, 66(2), 218-239.

9. Barac Karin and Kgobalale Nebbel Motubatse. (2009). Internal audit outsourcing practices in South Africa. African Journal of Business Management. Vol.3 (13), pp. 969-979,

10. Carey.P, Simnett.R, Tanewski.G, (2000). Voluntary demand for internal and external auditing by family business. Auditing: A Journal of Practice \& Theory. Vol. 19.

11. Chapman, C., (2001). Raising the bar. Internal Auditor. April, pp.55-59

12. Clark, M., Gibbs, T., \& Schroeder R., (1980). Evaluating internal audit departments under SAS No. 9: Criteria for judging competence, objectivity, and performance. The Woman CPA, Vol 2, 8-11.

13. Cohen, J., Krishnamoorthy, G., \& Wright A., (2004). The corporate governance mosaic and financial reporting quality. Journal of Accounting Literature, 23, 87-152.

14. Cooper, B. J., Leung, P., \& Wong G., (2006). The Asia Pacific literature review on internal auditing. Managerial Auditing Journal, 21(8), 822-834.

15. Coram, P., Ferguson, C., \& Moroney R., (2008). Internal audit, alternative internal audit structures and the level of misappropriation of assets fraud. Accounting \& Finance, 48(4), 543-559.

16. Deegan C. M., (2009). Financial accounting theory (3 ed.). North Ryde, NSW: McGraw-Hill.

17. DeZoort, F. T., Hermanson, D. R., Archambeault, D. S., \& Reed, S. A. (2002). Audit committee effectiveness: A synthesis of empirical audit committee literature. Journal of Accounting Literature, 21, 38-75.

18. Dirsmith, M. W., Fogarty, T. J., \& Gupta P., (2000). Institutional pressures and symbolic displays in a GAO context. Organization Studies, 21(3), 515-537.

19. Donna Jane Reed, (1999). Describing the demand for Internal Audit Services in U.S Colleges and Universities. Mississippi State University.

20. Elliott R.K, P Jacobson, (1992). Audit independence: Concept and application. The CPA Journal.

21. Engle, Terry (1999). Managing External Auditor Relationships. Internal Auditor, Academic One File, pp. 65.

22. Fadzil, F. H., Haron H., \& Jantan M., (2005). Internal auditing practices and internal control system. Managerial Auditing Journal, 20(8), 844 - 866.

23. Firth-Cozens, J. 1992. Building teams for effective audit. Quality in Health Care, 1, 252-255.

24. Freeman, (1984). Strategic Management: A Stakeholder Approach. Boston, MA: Pitman.

25. Fraser, I., and Henry W., (2007). Embedding risk management: structures and approaches. Managerial Auditing Journal. Vol. 22 No. 4, pp. 392-409

26. Goodwin, J. \& Kent, P. (2004). Factors affecting voluntary use of Internal Audit, Working Paper 2004-001, Queensland University of Technology.

27. Gramling, A. A., P. M. Myers, (1997). Practitioner' and users' perceptions of the benefits of certification of internal auditors. Accounting Horizons. Vol 11(1): 39-53

28. Gramling, A. A. and Myers, P. M., (2006), Internal Auditing's Role in ERM. Internal Auditor, Vol.63 No. 2, pp. 52-58. 
CÁC TRƯỜNG ĐẠI HỌC CÔNG LẬP THEO CƠ CHẾ TƯ CHỦ VIỆT NAM - NGHIÊN CÚU TİNH HUỐNG TẠI TRƯỜNG ĐẠI HỌC CÔNG NGHIỆP TP.HCM

29. Hair, J. F., Black, W. C., Babin, B. J. \& Anderson, R. E., 2010. Multivariate Data Analysis. 7th ed. s.1.:Pearson Printice Hall.

30. Hajiha Zohreh and Rafiee Azadeh, (2011). The Impact of Internal Audit Function Quality on Audit Delay, Middle-East Journal of Scientific Research, Vol.10 (3), 389-397.

31. Haron, H., Jantan, M., \& Pheng, E. G. (2005). Audit committee compliance with Kuala Lumpur Stock Exchange listing requirements. International Journal of Auditing, 9, 187-200.

32. Haron, H., Chambers, A., Ramsi, R., \& Ismail, I., (2004). The reliance of external auditors on internal auditors. Managerial Auditing Journal, 19(9), 1148-1159IIA, 2017. International Standards for the Professional Practice of Internal Auditing Guide: Independence and Objectivity. Altamonte Springs, Florida: the Institute of Internal Auditors.

33. Hass, S., Abdolmohammadi, M.J. and Burnaby, P. (2006). The Americas literature review on internal auditing. Managerial Auditing Journal. Vol. 21 No. 8, pp. 835-844.

34. Hoàng Trọng \& Chu Nguyễn Mộng Ngọc, (2008). Phân tích dĩ liệu nghiên cứu với SPSS. Nhà xuất bản Hồng Đức.

35. James và Mula, (2010). Antecedents and organisational performance implications of internal audit effectiveness: some propositions and research agenda. Pacific Accounting Review. Vol 22, Number 3, pp. 224-252(29)

36. Jensen, M. C., \& Meckling, W. H., (1976). Theory of the firm: Managerial behavior, agency costs and ownership structure. Journal of Financial Economics, 3(4), 305-360.

37. Joe Christopher, Gerrit Sarens, Philomena Leung (2009), A critical analysis of the independence of the internal audit function: evidence from Australia, Accounting, Auditing \& Accountability Journal, Vol. 22 (2), $200-220$.

38. Kalbers, L. P., and T. J. Fogarty. (1993). Audit committee effectiveness: An empirical investigation of the contribution of power. Auditing: A Journal of Practice \& Theory (Spring): 24-49.

39. Liu, J., Woo, H. \& Boakye-Bonsu, V. (1997). Developing Internal Auditing procedures using a benchmarking approach. Managerial Auditing Journal, Vol. 12, No. 9, pp. 464-78.

40. Mat Zain, M., \& Subramaniam N, (2007). Internal auditor perceptions on Audit Committee interactions: A qualitative study in Malaysian public corporations. Corporate Governance: An International Review, 15(5), 894-908.

41. Messier, W. F., Jr., and A. Schneider. (1988). A Hierarchical Approach to the External Auditor's Evaluation of the Internal Auditing Function. Contemporary Accounting Research 4 (2):337-353.

42. Nguyễn Đình Thọ và Nguyễn Thị Mai Trang. (2011). Nghiên cứu khoa học Marketing: úng dụng mô hình cấu trúc tuyến tính SEM. Nhà xuất bản Lao động, ấn bản lần 2, Việt Nam.

43. Nguyễn Phú Giang, (2015). Kiểm toán nội bộ hiện đại. Nhà xuất bản tài chính.

44. Powell S. F., (1993). Internal audit in industry. Management Accounting, 71(8), 52 \& 54.

45. Quales Ross, (1994). An Examination Of Promotion Opportunities And Evaluation Criteria As Mechanisms For Affecting Internal Auditor Commitment, Job Satisfaction And Turnover Intentions. Journal of Managerial Issues. Vol. 6, No. 2, pp. 176-194. 
46. Read J. William, Rama. D.V., (2003). Whistle-blowing to internal auditors. Managerial Auditing Journal. Vol. 18 Issue: 5, pp.354-362

47. Rose et al, (2013). Is the objectivity of internal audit compromised when the internal audit function is a management training ground? Accounting and Finance.

48. Sarbanes-Oxley (2002). Sarbanes-Oxley Act of 2002. One Hundred Seventh Congress of the United States of America, HR 3763.

49. Sarens, G., \& De Beelde I., (2006). The relationship between internal audit and senior management: A qualitative analysis of expectations and perceptions. international journal of Auditing, 10(3), 219-241.

50. Sawyer, L.B. (1988). Sawyers' Internal Auditing, Institute of Internal Auditors, Altamonte Springs, FL.

51. Spence M, (1973). Job Market Signaling. The Quarterly Journal of Economics, Vol. 87, No. 3. (Aug., 1973), pp. 355-374.

52. Spraakman, G. (2001). Internal audit at the historical Hudson's bay company: A challenge to accepted history. Accounting Historians Journal(June): 19- 41

53. Vanasco, R. R., (1994). The audit committee: an international perspective. Managerial Auditing Journal, 9(8), $18-42$.

54. Van Staden, M., and B. Steyn., (2009). The profile of the chief audit executive as a driver of internal audit quality. African Journal of Business Management 3: 918-925.

55. Yee, C. S., Sujan, A., James, K. \& Leung, J. K.-S., (2008). The Perception of the Singaporean Internal Audit Customers Regarding the Role and Effectiveness of Internal Audit. Asian Journal of Business and Accounting. Vol. 1 No. 2, pp. 147-174.

56. Watts R.L \& Zimmerman, J., (1983). Agency problems, auditing, anh the theory of the firm: some evidence. Journal of Law and Economic. Vol 26, pp 613-634.

Ngày nhận bài: 14/05/2018

Ngày chấp nhận đăng: 10/12/2018 